\title{
Patterns of spontaneous activity in unstructured and minimally structured spinal networks in culture
}

Received: 8 July 2004 / Accepted: 20 January 2005 / Published online: 7 June 2005

(C) Springer-Verlag 2005

\begin{abstract}
The rhythmic activity observed in locomotion is generated by local neuronal networks in the spinal cord. The alternating patterns are produced by reciprocal connections between these networks. Synchronous rhythmic activity, but not alternation, can be reproduced in disinhibited networks of dissociated spinal neurons of rats. This suggests that a specific network architecture is required for pattern generation but not for rhythm generation. Here we were interested in the recruitment of neurons to produce population bursts in unstructured and minimally structured cultures of rat spinal cord grown on multielectrode arrays. We tested whether two networks, connected by a small number of axons, could be functionally separated into two units and generate more complex patterns such as alternation. In the unstructured cultures, we found that the recruitment of the neurons into bursting populations is divided into two steps: the fast recruitment of a "trigger network", consisting of intrinsically firing cells connected in networks with short delays, and slow recruitment of the rest of the network. One or several trigger networks were observed in a single culture and could account for variable patterns of propagation. In the minimally structured cultures, a functional separation between loosely connected networks was achieved. Such separation led either to an independent bursting between the networks or to synchronized bursting with long and variable delays. However, no qualitatively novel pattern such as alternation could be generated. In addition, we found that the strength of reciprocal inhibitory connections was modulated by spontaneous activity.
\end{abstract}

C. Yvon $(\bowtie) \cdot$ R. Rubli $\cdot$ J. Streit

Department of Physiology,

Physiologisches Institut,

University of Bern, Bühlplatz 5,

3012 Bern, Switzerland

E-mail: yvon@pyl.unibe.ch

Tel.: + 41-31-6318749

Fax: +41-31-6314611
Keywords Neuronal network · Bursting ·

Multielectrode array $\cdot$ Glutamate

\section{Introduction}

Repetitive rhythmic activity is a feature of neural assemblies, which has been attributed to key functions of the central nervous system (Grillner 2003; Steriade 2001). While such rhythm generation often requires well-structured networks of excitatory and inhibitory interneurons (Whittington and Traub 2003), it can also be based on repetitive activation of a network through recurrent excitatory connections leading to rhythmic population bursting. Such rhythms have been described in cortical (Sanchez-Vives and McCormick 2000), hippocampal (Staley et al. 1998) and brain stem slices (Rekling and Feldman 1998), as well as in spinal slice cultures (Ballerini et al. 1999; Tscherter et al. 2001). They require, first, intrinsically spiking cells, which are able to recruit the network and, second, one or several accommodation mechanisms to calm down the network (Darbon et al. 2002, 2003). However, they do not require a specific architecture in terms of inhibitory and excitatory connections, since rhythmic activity can appear most reliably after blockade of synaptic inhibition (disinhibition). Furthermore, such rhythms can also be produced in networks of dissociated neurons, which are entirely grown in vitro (Droge et al. 1986; Muller and Swandulla 1995; Robinson et al. 1993; Streit et al. 2001).

More complex patterns of activity, such as alternating rhythms, which are used as output for locomotion, require more complex networks. Such networks do not spontaneously grow in vitro, neither in dissociated cultures nor in slice cultures (Streit et al. 2001; Tscherter et al. 2001). The reason for this is that, for example, alternation between the left and the right side in the spinal cord needs a subtle balance between excitatory and inhibitory commissural connections, which cross 
from one side to the other (Beato and Nistri 1999). Recently it has been shown that this balance may be achieved through specific inhibitory actions by the ephrin/Eph system on axons which are growing towards the midline (Kullander et al. 2003). Such structures are usually not maintained in culture. Nevertheless, attempts have been made in recent years to structure neural networks that are grown in vitro. This was achieved either by growing the cells on patterned substrates (Rohr et al. 1999) or in compartments (Fields et al. 1991). Using these approaches, structured networks can be grown in vitro and the functional consequences of such structuring can be investigated.

In this work, we have used unstructured and minimally structured cultures, which were grown on multielectrode arrays (MEAs) to investigate the recruitment of cells to produce population bursts. We were interested in how activity spreads in two to four networks connected by a small number of axons and whether a sufficient delay may be produced between the networks to produce stable alternation in bursting. Such stable phase shifts were predicted in a theoretical study for loosely coupled local oscillating networks (Senn et al. 1998). We found that, although long delays appeared between such networks, no stable alternation in bursting occurred.

\section{Materials and methods}

\section{Culture preparation}

All cultures were made from the spinal cord of rats at embryonic age 14. The cultures were prepared as described previously (Streit et al. 2001). The embryos were delivered by Caesarian section from deeply anaesthetized animals ( $0.4 \mathrm{ml}$ pentobarbital i.m.) and euthanased by decapitation. Following the delivery of the embryos, the mother was euthanased by intracardiac injection of pentobarbital. Animal care was in accordance with guidelines approved by Swiss local authorities. The backs of the embryos were isolated from their limbs and viscera and cut into $225-\mu \mathrm{m}$ thick transverse slices with a tissue chopper. For the dissociation of the cells, slices of all regions of the spinal cord, without dorsal root ganglia, were exposed to a $0.3 \%$ trypsin solution for $3 \mathrm{~min}$ at $37^{\circ} \mathrm{C}$. These slices were then mechanically dissociated by forcing them through fine-tipped pipettes several times. The cells were plated on MEAs at a density of $150,000 / 150 \mu$ l. The MEAs were produced as described previously (Tscherter et al. 2001) and coated for $1 \mathrm{~h}$ with diluted (1:50) Matrigel (Falcon/Biocoat, Becton Dickinson, Switzerland). They were maintained in culture dishes containing nutrient medium and incubated in a $5 \% \quad \mathrm{CO}_{2} / 95 \%$ air atmosphere at $36.5^{\circ} \mathrm{C}$ for up to 12 weeks. Serum-free Neurobasal medium (Gibco BRL, Life Technologies, Switzerland) supplemented with B27 and Glutamax (both Gibco BRL) was used and half of the medium was changed twice a week.
Unstructured and minimally structured cultures

For unstructured cultures, the cells were-as described previously (Streit et al. 2001) - restricted to an area around the electrodes (ca. $50 \mathrm{~mm}^{2}$ ) using cloning glass cylinders attached to the MEAs by silicone sealant. Within the area defined by the cloning tube, the cells formed a random network on the homogeneously coated surface. The cylinders were filled with $150 \mu \mathrm{l}$ of nutrient medium. Minimal structure was imposed on the cultures by forcing the cells to form networks on four separated compartments. This was achieved by using plexiglas masks having four equally sized and spaced holes $(4 \mathrm{~mm}$ in diameter, $7.2 \mathrm{~mm}$ apart from each other). These masks were attached by silicone sealant to MEA dishes coated with Matrigel, on which the electrodes were grouped in four hexagonal zones of 17 electrodes each. The cells were seeded in each of the four holes and covered with $80 \mu \mathrm{l}$ of nutrient medium each. After 5 days, the masks were removed, thus allowing some axons to grow out from the networks in the four compartments and to establish connections between the compartments. Although there were no growth restrictions, usually only a few axons crossed the areas between the compartments. Therefore, this procedure produced cultures consisting of four separate compartments, which could be connected by a few axons or bundles of axons (see Fig. 5b). Such compartment cultures were investigated between 2 and 10 weeks after the mask had been removed.

\section{Recordings}

Recordings were made in a chamber mounted on an inverted microscope (Nikon, Japan) from cultures of 4 12 weeks of in vitro age. The medium was replaced by an extracellular solution containing (in $\mathrm{mM}$ ): $\mathrm{NaCl}, 145$; $\mathrm{KCl}, 4 ; \mathrm{MgCl}_{2}, 1 ; \mathrm{CaCl}_{2}, 2$; HEPES, 5; Na-pyruvate, 2; glucose, 5 at $\mathrm{pH}$ 7.4. Recordings were either made in the presence of continuous superfusion at $1 \mathrm{ml} / \mathrm{min}$ or with solution changes every 10-15 min. No differences in the patterns of activity were seen between these two protocols. All recordings were made at room temperature (22$\left.26^{\circ} \mathrm{C}\right)$.

\section{MEA recording and analysis}

The MEAs contained 68 electrodes, laid out in the form of a rectangle (unstructured cultures) or of four hexagons (compartment cultures). Channels showing activity (usually 10-40) were selected by eye and their recordings digitized at $6 \mathrm{kHz}$, visualized and stored on hard disk using custom-made virtual instruments within Labview (National Instruments, Switzerland), as described previously (Tscherter et al. 2001). Detection of the extracellularly recorded action potentials and further analysis were done offline in the software package IGOR 
(WaveMetrics, Lake Oswego, Ore., USA) as described previously. The electrodes recorded fast voltage transients (see Fig. 1a), which correspond to single action potentials in neurons or axons (single-unit activity). These fast voltage transients often appeared in clusters (multi-unit activity) originating from closely timed action potentials ( $<4 \mathrm{~ms}$ apart) of several neurons seen by one electrode. During such multi-unit activity, individual fast voltage transients could not be clearly linked to underlying action potentials and therefore activity was set to a defined level of $333 \mathrm{~Hz}$ (= the upper limit of temporal resolution of the detector). Thus the event detector reliably translated single-unit activity into single events and also multi-unit activity into trains of events defined at $333 \mathrm{~Hz}$ (Tscherter et al. 2001). No attempt was made to sort spikes seen by one electrode. The selectivity of event detection was controlled using recordings obtained in the presence of tetrodotoxin (TTX, $1.5 \mu \mathrm{M})$ as a zero reference. The processed data were displayed in the form of event raster plots and network activity plots (see Fig. 1b). Event raster plots show the time-markers of detected activity of each selected channel (e.g., Fig. 1a). Network activity plots (e.g., Fig. 1b) show the total activity of all selected channels within a sliding time window of $10 \mathrm{~ms}$, shifted by $1 \mathrm{~ms}$ steps. Activity delays between electrodes were either measured from the event raster plots at the onset

Fig. 1a-d MEA recording from networks of dissociated spinal neurons. a Three original traces of extracellular recordings from three electrodes reveal a population burst. b Raster plot showing the time markers of activity at nine electrodes (below), together with the network activity plot showing the integrated activity over all electrodes (above). The numbers refer to the three traces shown in a. c Distribution of activity in the culture. The diameter of the white circles is proportional to the amount of activity seen by that electrode. d Delays in the burst onset at each electrode compared with the burst start. The burst source is the electrode on the left with a delay of 0 . The lines are isochrones, which are extrapolated from the delays of bursts or directly from the original recordings. The delays corresponding to the recruitment of more and more neurons in the network were in the range of 20 to several hundred milliseconds and could therefore be well resolved from the event rasters, although the temporal resolution of event detection was low $(3 \mathrm{~ms})$. However, the delays corresponding to axonal conduction were in the range below $5 \mathrm{~ms}$. They were therefore measured from averages of time-windows of several original traces, which were triggered by the same events (see Fig. 4).

\section{Whole-cell patch clamp recording and analysis}

Intracellular voltage was obtained from individual neurons in cultures on MEAs using the whole-cell patch technique (Hamill et al. 1981) with an Axoclamp 2B amplifier (Axon Instruments, Union City, Calif., USA). The patch pipettes were filled with a solution containing (in $\mathrm{mM}$ ): K-gluconate, $100 ; \mathrm{KCl}, 20 ;$ HEPES, $10 ; \mathrm{Mg}$ ATP, 4; $\mathrm{Na}_{2}$-GTP, 0.3; $\mathrm{Na}_{2}$-Phosphocreatine, 10 ; pH 7.3 (with $\mathrm{KOH}$ ). The electrodes had a resistance of 4-5 M $\Omega$. No series resistance compensation was applied. Native resting membrane potentials were in the range of -40 to $-60 \mathrm{mV}$. Cells with a potential less negative than $-40 \mathrm{mV}$ were discarded. Voltage recordings were digitized, visualized and stored on computer using custommade virtual instruments within Labview (National Instruments, Switzerland). They were analyzed offline using custom-made programs in IGOR (WaveMetrics).

\section{Stimulation by MEA electrodes}

Biphasic monopolar voltage pulses with a duration of $0.5 \mathrm{~ms}$ and amplitudes of $0.5-1.25 \mathrm{~V}$ were addressed to one of the MEA electrodes. No recording was possible with the electrode connected to the stimulator.
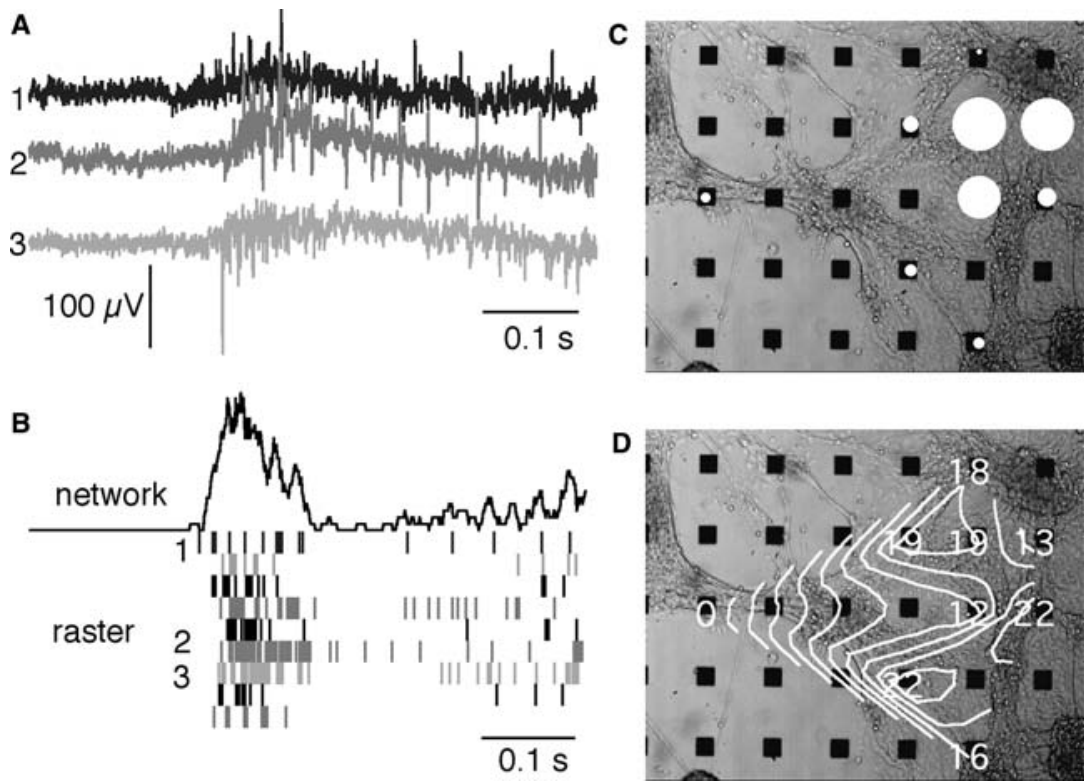


\section{Statistics}

Averages are expressed as mean \pm standard error of the mean (SEM). Statistics are based on Student's $t$-test and the Wilcoxon test.

\section{Chemicals}

All agents (APV, bicuculline, CNQX, nipecotic acid, NMDA, sarcosine, strychnine and TTX, all Sigma) were bath applied.

\section{Results}

Experiments were made from 19 unstructured cultures and 38 compartment cultures with an in vitro (DIV) age of 22-51 days. Within this period, the cultures had reached a steady state in terms of parameters describing network activity such as burst rate, burst duration and total activity. As previously described (Streit et al. 2001), all cultures showed spontaneous activity (see Fig. 1), which consisted of short bursts or waves of activity seen by most of the electrodes simultaneously. Usually these waves appeared irregularly with silent intervals between them. During these silent intervals, low rate activity appeared at one or a few electrodes, which did not spread to the whole network (asynchronous background activity). As shown in Fig. 1c, the total amount of activity seen by an electrode varied considerably among the different electrodes. Furthermore, we have shown previously that in unstructured cultures activity could start from several locations in the network (called burst sources) and spread from there to the whole network on variable pathways. Burst sources were defined as electrodes at which bursts started more than once. The spread of activity was displayed as shown in Fig. 1d by measuring, for each burst, the delays of the onset of activity at all electrodes compared with the source. From such a distribution of delays, activation isochrones were extrapolated. In the experiment shown in Fig. 1, the activation of the part of the network measured and displayed in D took $22 \mathrm{~ms}$. This was short compared with the usual activation time of 60-120 ms (compare Legrand et al. 2004; Streit et al. 2001). In some cultures parts of the networks even took $150-200 \mathrm{~ms}$ to be recruited into the ensemble activity (see Fig. 2a). To look for mechanisms involved in such a high variability of delays, we first investigated burst onsets in unstructured cultures.

\section{Trigger networks}

Stable and reliable bursting was induced by disinhibition of the cultures through the application of strychnine $(1 \mu \mathrm{M})$ to block the glycine conductances and bicuculline $(20 \mu \mathrm{M})$ to block the $\mathrm{GABA}_{\mathrm{A}}$ conductances. As previously reported (Streit et al. 2001) and shown in Fig. 2a, long-lasting (1-4 s) bursts of population activity appear at more or less regular rates (2-6 bursts/min) under such conditions. Such population bursts usually originate from several burst sources. In Fig. 2a, the spread of activity is shown for four different bursts. All four originate from different sources (indicated by black arrows). Nevertheless it is evident that the global pattern of the isochrones is similar for the first three bursts indicated (1-3), whereas it seems to be completely different in the last burst (4). In order to quantify the similarity between two patterns, we plotted the delays at all electrodes of one burst versus another. Figure $2 b$ shows three of such plots, which clearly show that a highly significant correlation was seen for burst 2 versus 1 and for burst 3 versus 1 , but not for burst 4 versus 1 . These findings show that some of the burst sources are connected in networks with short delays. We call such networks "trigger networks". We then determined the number of trigger networks per culture by grouping the bursts into several pools. Each pool contained all the bursts, which were linked by a significant correlation of delays as shown in Fig. 2b. In the culture shown in Fig. 2, two such pools (= trigger networks) were counted. In a sample of 11 cultures, $1-5$ trigger networks were found per culture, with a mean value of $1.9 \pm 0.37$. Five of these cultures contained only one trigger network. The mean number of burst sources in this sample was $4.4 \pm 0.5$, corresponding to $31.4 \pm 3.8 \%$ of all electrodes that recorded activity, and the mean delay was $61.2 \pm 6.4 \mathrm{~ms}$. All these values agreed well with those established in previous studies (Legrand et al. 2004; Streit et al. 2001).

Next we examined whether the electrodes which recorded high activity rates also had short delays. To do this, we determined the correlation between the delays and the total activity (measured in disinhibited cultures). As shown in Fig. 2c, a negative correlation was found. The mean correlation coefficient $r$ was $-0.61 \pm 0.09$ in 11 experiments. It reached significance in eight of these experiments. We then investigated the correlation between the same delays (measured at the onset of population bursts during disinhibition) and the intrinsic activity (measured in the presence of strychnine, bicuculline and the glutamate receptor blockers CNQX and APV; see also Darbon et al. 2002; Streit et al. 2001). In this case we also found a negative but weaker correlation (see Fig. 2d). The mean $r$ was $-0.45 \pm 0.1$ in nine experiments, reaching significance in four of these experiments. We conclude from these findings that the trigger networks are formed by highly excitable cells, which are at least partly intrinsically spiking.

While population bursting induced by disinhibition is slow and not always regular, it can be turned into a fast and highly regular rhythm by NMDA (Legrand et al. 2004). Such a rhythm is additionally characterized by smaller activity within the bursts, more activity in the intervals and a slower burst onset. However, in spite of the highly regular rhythms, the variability in burst 
Fig. 2a-d Network recruitment in disinhibition induced bursting. a Recruitment delays (numbers in milliseconds) and isochrones (lines) showing the pathways of network recruitment for four different bursts with different sources (indicated by black arrows). The network activity trace is shown above. Note the difference in the general pathways between 1 to 3 on one hand and 4 on the other. b Plots of the delays in one burst versus another. A significant correlation was found for 2 versus 1 and for 3 versus 1 , but not for 4 versus 1 . c Plot of the mean delays for each electrode (over all bursts) versus the mean overall activity during bursting at each electrode. d Plot of the mean delays for each electrode (over all bursts) versus the mean intrinsic activity after blockade of fast synaptic transmission (by bicuculline, strychnine, CNQX and APV) at each electrode
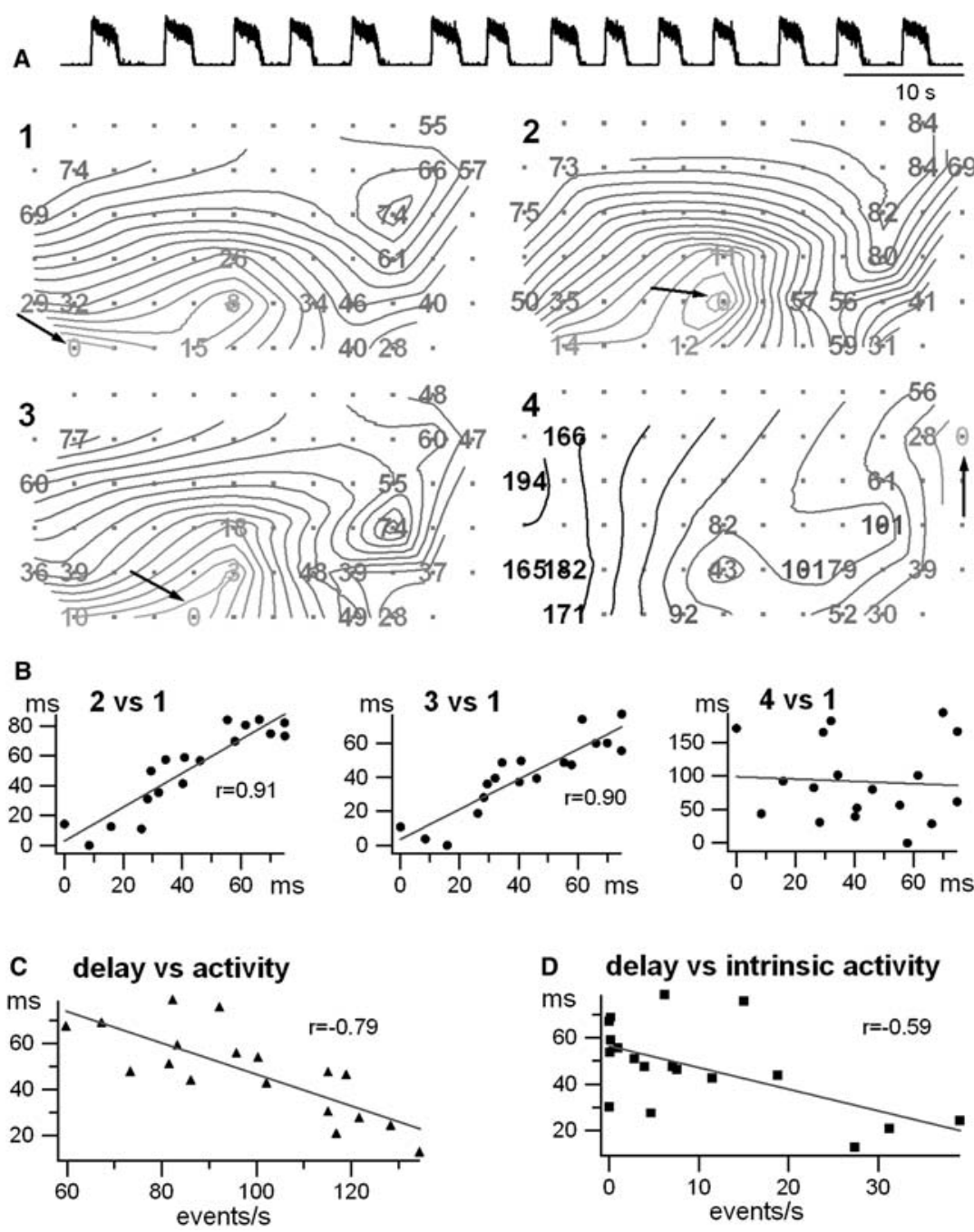

sources and propagation was not changed with NMDA. One possible explanation for these somehow contradictory findings is that such NMDA rhythms are controlled by one single trigger network per culture. Indeed, we found only one trigger network when we did the correlation analysis in seven cultures treated with strychnine, bicuculline and NMDA. This in spite of the fact that the mean number of burst sources $(6.6 \pm 0.7$ corresponding to $43.1 \pm 4.9 \%$ of all active electrodes) was even higher than during disinhibition alone.

As shown previously (Legrand et al. 2004), NMDA, by depolarizing the neurons, causes an increase in the amount of intrinsic spiking as well as an improved synaptic coupling of intrinsically spiking cells to the network. To find out which of these two mechanisms is more important in defining a burst source, we plotted the number of burst sources and the number of intrinsically active cells versus the network size in different cultures. As a measure of network size, we took the total number of electrodes per culture, which recorded activity. As a measure of intrinsically active cells, we took the number of electrodes recording activity after blockade of synaptic transmission (in the presence of strychnine, bicuculline, APV and CNQX). As shown in Fig. 3a, b, we found no correlation between the number of burst sources and the network size, whereas the number of intrinsically spiking cells was clearly correlated to network size $(r=0.72, P<0.01)$. This finding shows that while the number of intrinsically spiking cells increases proportionally with increasing network size, the number of burst sources is independent of network size. We conclude from this finding that burst sources are not mainly defined by the number of intrinsically spiking cells but much more by the question of how many of these cells are able to trigger a population burst in the network through their synaptic contacts (see also the discussion). In order to get an idea of the percentage of the cells, which have this capability, we evoked spikes in individual neurons by intracellular current injection during whole-cell recordings and measured the network response. In 14 out of 15 such experiments the network did not respond to the activation of a single cell (see Fig. 3c). In one neuron, spikes evoked by depolarizing current pulses reliably triggered network responses (in 33 of 33 pulses, see Fig. 3d). This finding confirms our hypothesis that there are neurons in the culture, which 


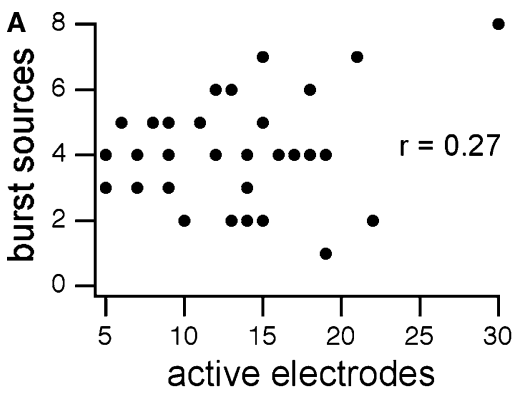

C

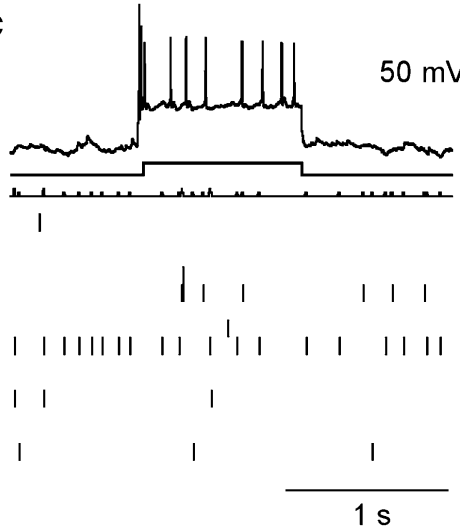

Fig. 3a-d Burst sources and intrinsic activity. a Plot of the burst sources $(=$ the number of electrodes where more than one burst started during disinhibition by $\mathrm{B} / \mathrm{S}$ ) versus the active electrodes (= the total number of electrodes which recorded activity during disinhibition by $\mathrm{B} / \mathrm{S}$ ). Each point represents one culture. b Plot of the intrinsic electrodes ( $=$ the number of electrodes which recorded activity under bicuculline, strychnine, APV and CNQX) versus the active electrodes $(=$ the total number of electrodes which recorded activity during disinhibition by $\mathrm{B} / \mathrm{S}$ ). Each triangle represents one culture. c Intracellular whole-cell recording from one neuron (above) in combination with the network activity and the raster plot from MEA recordings of the same culture (below). Note that the spikes which were evoked in the neuron by injection of a depolarizing current pulse did not evoke a response of the network. d Intracellular whole-cell recording from one neuron (above) in combination with the network activity and the raster plot from MEA recordings of the same culture (below). Note that the spikes which were evoked in the neuron by injection of a depolarizing current pulse evoked a clear network response. (Different culture than in $\mathbf{c})$

are able to trigger population bursts, but that they are rare.

\section{Axonal conduction velocity}

The findings presented so far suggest that the recruitment of the neurons into population bursts occurs in two steps: first, the fast recruitment of the cells belonging to the trigger network (or networks) and then the slower recruitment of the rest of the network. Within this hypothesis the delays of the trigger network may be largely influenced by the axonal conduction velocity and the distances between the cells. In order to test this assumption experimentally, we measured conduction velocity in our cultures. To do this we took advantage of the finding that occasionally a very strong temporal correlation
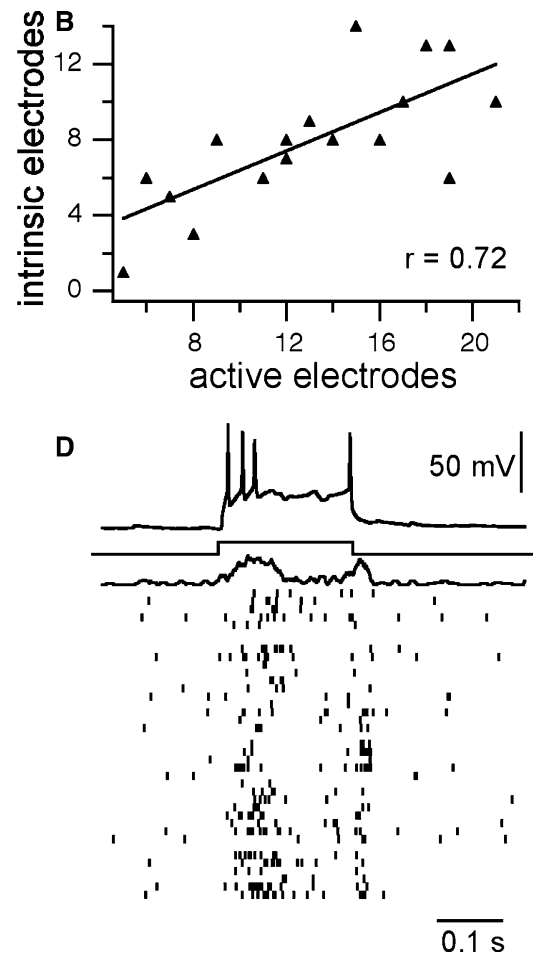

occurred between the signals of two electrodes. Figure 4 shows an example in which such a correlation even occurred between four electrodes. This synchrony of signals occurred in a time-window of a few milliseconds and was resistant to blockers of synaptic transmission (strychnine, bicuculline, CNQX and APV, experiments not shown), thus suggesting that the signals originated from the same axon, which crossed several electrodes. Thus, the mean delay between the signals could be used to estimate axonal conduction velocity. The mean delays were measured from averages of the original recordings from the electrodes, which were triggered by the events detected in one of these recordings. In the experiment shown in Fig. 4, a conduction velocity of $0.3 \mathrm{~m} / \mathrm{s}$ was calculated from the plot of the distance between the electrodes versus the delays. From seven such experiments (usually with a synchrony between the signals of two electrodes) a mean axonal conduction velocity of $0.35 \pm 0.06 \mathrm{~m} / \mathrm{s}$ was calculated. The conduction time for a distance of $2.2 \mathrm{~mm}$ (corresponding to the length of the array) would thus be $6.3 \mathrm{~ms}$, a number, which is in the range of the shortest delays measured between two remote electrodes (around $10 \mathrm{~ms}$ ). The tenfold higher network recruitment times of 60-120 ms usually seen are therefore not mainly caused by the axonal conduction velocity.

\section{Coupling in compartment cultures}

To investigate how spatial heterogeneity in the network influences the recruitment of neurons into population bursts, we measured the spread of activity during the onset of bursts in compartment cultures. We were interested in whether such a minimal structure could 
Fig. 4 Axonal conduction velocity. Raster plot on the upper left side shows two groups of highly synchronous channels. Averages of the original traces of the marked channels in time windows, which are triggered by the time markers in one of these channels ("spike triggered averages") are shown on the upper right side. The involved electrodes are shown on the lower left side and the plot of the distances versus the delays on the lower right side. The slope of the linear regression line $(0.3 \mathrm{~m} / \mathrm{s})$ is the axonal conduction velocity
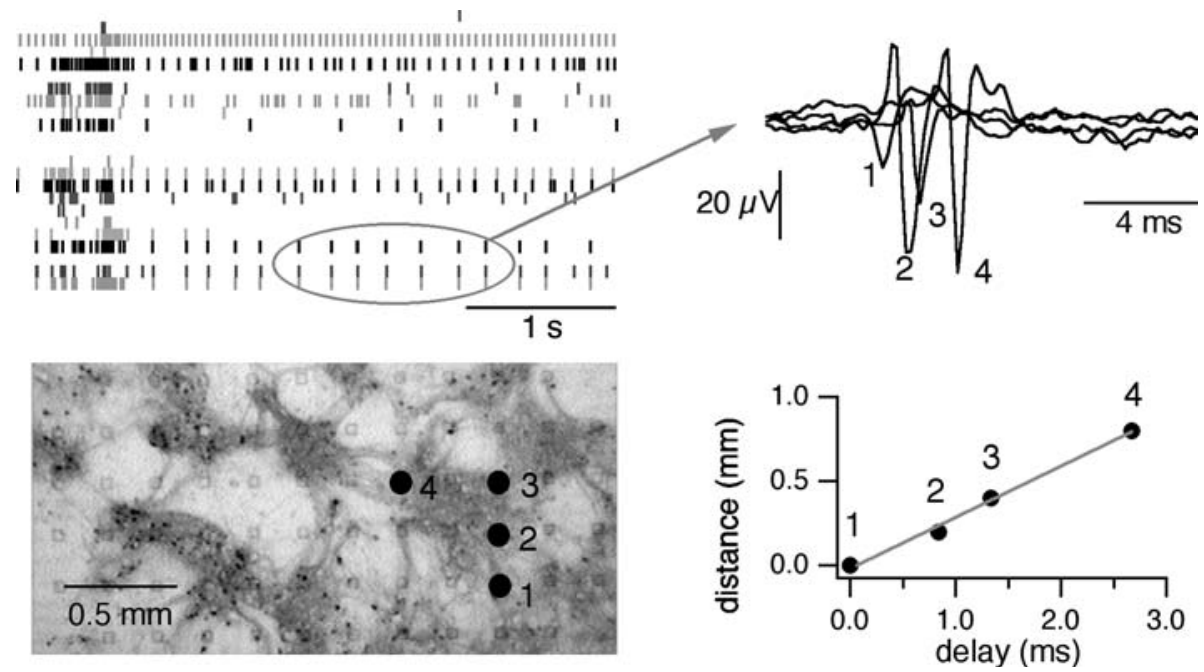

functionally separate the networks into functional units and thus serve as a mechanism to generate more complex patterns than synchrony. Indeed, it has been proposed in a theoretical study that loosely coupled excitatory networks may generate patterns of activity with phase lags, such as, for example, alternation (Senn et al. 1998). In order to test this hypothesis experimentally, we developed a minimally structured culture system, in which the coupling between two local networks was just sufficient to obtain interactions. Such a system was obtained by growing four separate networks in a dish during the first 5 days in culture, which were allowed to innervate each other in a subsequent period. Figure $5 \mathrm{~b}$ shows a compartment culture after 5 weeks in vitro. In $90 \%$ of such cultures coupling between the compartments was morphologically evident by single axons and small bundles of axons crossing the area between the compartments. Depending on the culture, this was true for two, three or all four compartments (as shown in Fig. 5b). Functional coupling was seen by the correlation of the beginning of the bursts in different compartments and thus quantified in cross-correlograms between two compartments. Figure 5 shows the correlation of bursts (Fig. 5a, c, d) and the corresponding cross-correlograms (Fig. 5e) in one experiment performed under disinhibition. In this example, coupling was seen between two pairs of compartments $(1,2$ and 3,4$)$, but not among the others. If all four compartments had developed in a culture, a maximum of six connected pairs would have been possible. Each pair could be connected in two directions. An average of $44 \%$ connections per culture (corresponding to 148 of a total of 334) were realized in a sample of 33 cultures that were investigated under disinhibition. Coupling between bursts was usually not $100 \%$ reliable. The mean delays between bursts in neighboring compartments, as measured from the position of the peaks of the cross-correlograms, was $250 \pm 42 \mathrm{~ms}$ under disinhibition $(n=12)$. However, delays were highly variable from burst to burst in individual experiments. Again they were about ten-times larger than expected from the axonal conduction velocity, which predicts a conduction time of about $21 \mathrm{~ms}$ for the distance of $7.2 \mathrm{~mm}$ between the compartments. Coupling between compartments was mainly mediated by AMPA/kainate receptors, since it could be blocked by CNQX at concentrations (5$10 \mu \mathrm{M}$ ), which did not yet suppress bursting in the compartments. At low concentration $(1 \mu \mathrm{M})$, CNQX increased the mean delay to $580 \mathrm{~ms}$ under disinhibition ( 3 cultures, $n=10$ connections, $P=0.0014$ ). In contrast, the NMDA blocker APV had neither an effect on coupling rate nor on the mean delays.

Obviously these delays in transmission were not sufficiently long to produce alternating patterns of bursting in two adjacent compartments, since the disinhibitioninduced population bursting with an average of one burst every $13 \mathrm{~s}$ was much too slow. However, even when the bursting was accelerated by NMDA with, on average, one burst every $5 \mathrm{~s}$ (Legrand et al. 2004), no stable phase shifts between the oscillations in neighboring compartments developed, although the mean delay was prolonged ( $369 \pm 58 \mathrm{~ms}, n=10$ cultures). We thus conclude from these experiments that such burst delays between minimally coupled excitatory networks cannot account for stable phase shifts in coupled oscillator networks.

Alternating activity in central pattern generators has been shown to depend critically on crossing inhibitory connections (Kullander et al. 2003). In order to test whether sufficient inhibitory coupling develops between compartments to evoke alternating patterns, we investigated bursting in the presence of inhibition. As previously reported by Streit et al. (2001), bursts appeared irregularly in control conditions $\left(4 \mathrm{mM} \mathrm{K} \mathrm{K}^{+}, 1 \mathrm{mM}\right.$ $\mathrm{Mg}^{2+}$, Fig. 6a). However, the rhythm could be made more regular in conditions of increased excitability (low $\mathrm{Mg}^{2+}$ /high K${ }^{+}$, Fig. 6b). Under both conditions the mean coupling rate was significantly smaller $(24 \%$, $n=12$ cultures, $P<0.002$ for $4 \mathrm{mM} \mathrm{K}{ }^{+}, 1 \mathrm{mM} \mathrm{Mg}^{2+}$ and $19 \%, n=18$ cultures, $P<0.002$ for low $\mathrm{Mg}^{2+} /$ high $\mathrm{K}^{+}$) compared with disinhibition, suggesting that inhibitory axons did indeed cross between compart- 

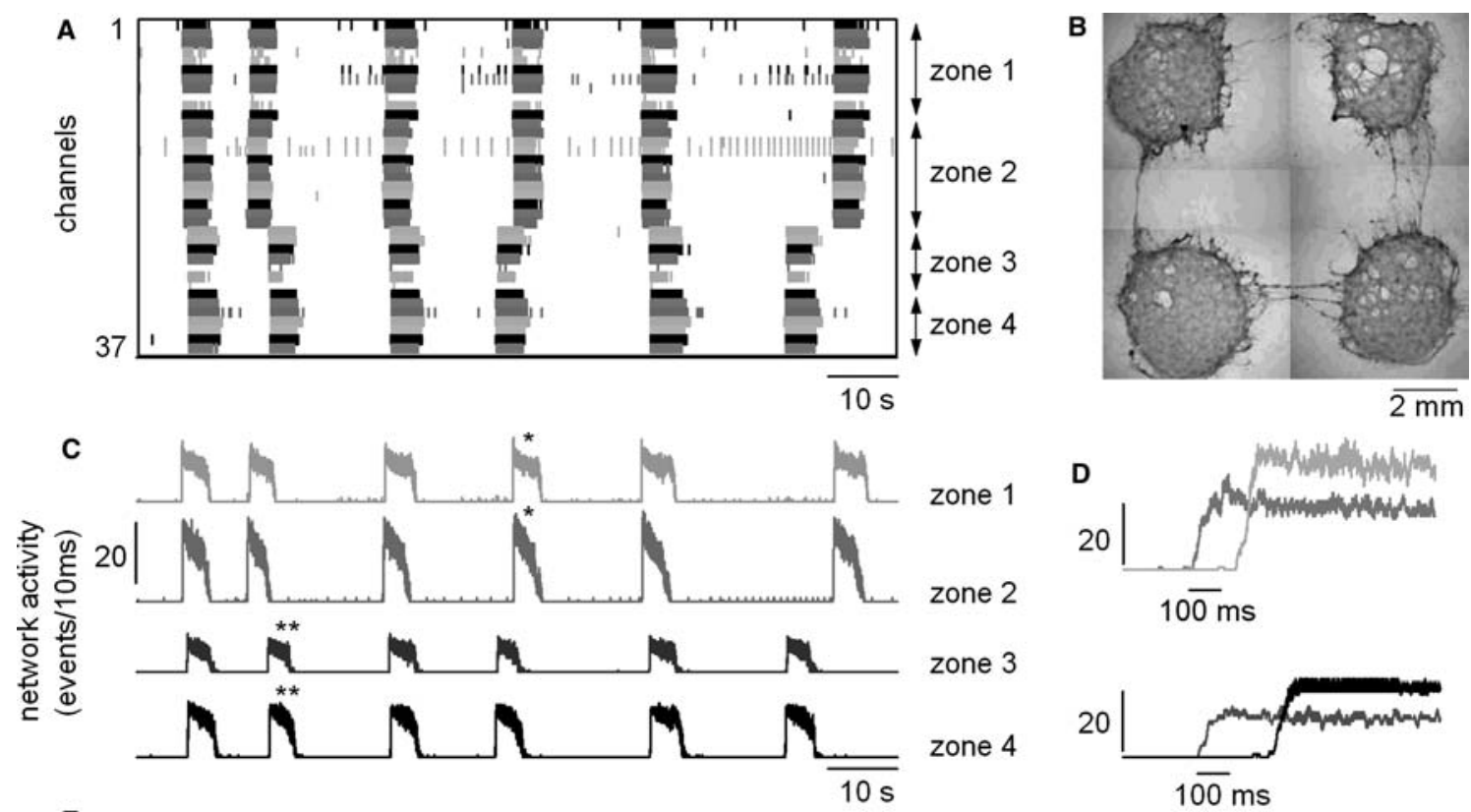
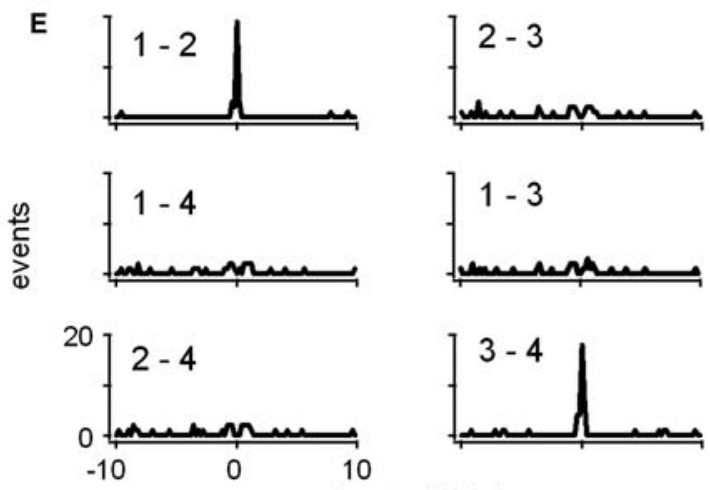

burst shift (s)

Fig. 5 Correlation of bursts in compartment cultures under disinhibition. a Event raster plot showing the time markers of 36 electrodes. Electrodes 1-11 belong to the first zone, electrodes 1223 belong to zone 2, electrodes 24-29 to zone 3 and electrodes 30 37 to zone 4. b Example of compartment cultures grown on an MEA dish after 5 weeks in vitro. Each culture lies on 17 electrodes arranged in a hexagonal form (electrodes are not visible here). Distance between nearby cultures ranges between 4 and $6 \mathrm{~mm}$. In this example, axons and small bundles of axons connect the four nearby compartments. c Separate network activity plots of the four zones showing the coupling between zones 1, 2 and 3, 4. d Enlarged and superimposed bursts which are correlated and marked by stars in c. Note the delays between the bursts, which were highly variable from burst to burst. e Cross-correlograms between compartments showing the coupling between the first two zones and the last two zones. f Enlarged cross-correlogram from a similar experiment showing two peaks near zero, one on the left and one on the right. This indicates that the connections between two compartments were present in both directions

ments. However, these inhibitory connections were not sufficient to produce alternating patterns, since the delays in bursting between two compartments were too short compared with the bursting period in the control condition $(170 \pm 21 \mathrm{~ms}, n=18$ vs $5.82 \pm 0.85 \mathrm{~s}, n=12$, see Fig. 6b) and in low $\mathrm{Mg}^{2+} /$ high $\mathrm{K}^{+}(138 \pm 18 \mathrm{~ms}$, $n=9$ vs $2.24 \pm 0.11 \mathrm{~s}, n=15$, see Fig. $6 \mathrm{~d}$ ). The delays in

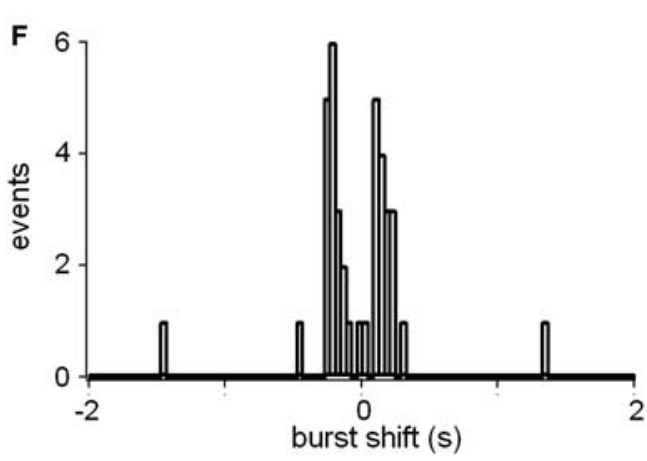

low $\mathrm{Mg}^{2+} /$ high $\mathrm{K}^{+}$were significantly shorter than under disinhibition $(-29 \pm 9 \%, n=7, P=0.014)$.

Strengthening inhibition by glycine re-uptake blockers in the isolated spinal cord of transgenic mice with too strong commissural excitation has been demonstrated to restore alternation between the left and the right side (Kullander et al. 2003). To test whether this is also true in the compartment cultures, we used the glycine and GABA $_{A}$ uptake blockers sarcosine $(100 \mu \mathrm{M})$ and nipecodic acid $(100 \mu \mathrm{M})$ to investigate their effects on rhythmic activity, which was induced by low $\mathrm{Mg}^{2+} /$ high $\mathrm{K}^{+}$. However, these drugs neither induced alternating rhythms between compartments nor had an effect on the mean delay or the coupling rate. They significantly decreased the burst rate $(-29 \pm 4 \%, n=5, P=0.0012)$, prolonged the intervals between the bursts $(+85 \pm 14 \%$, $n=5, P=0.0032)$ and increased the spike rate during the bursts $(+29 \pm 7 \%, n=5, P=0.012)$.

Activity-dependent modulation of coupling between compartments?

We next investigated the hypothesis that the strength and number of synaptic connections between 


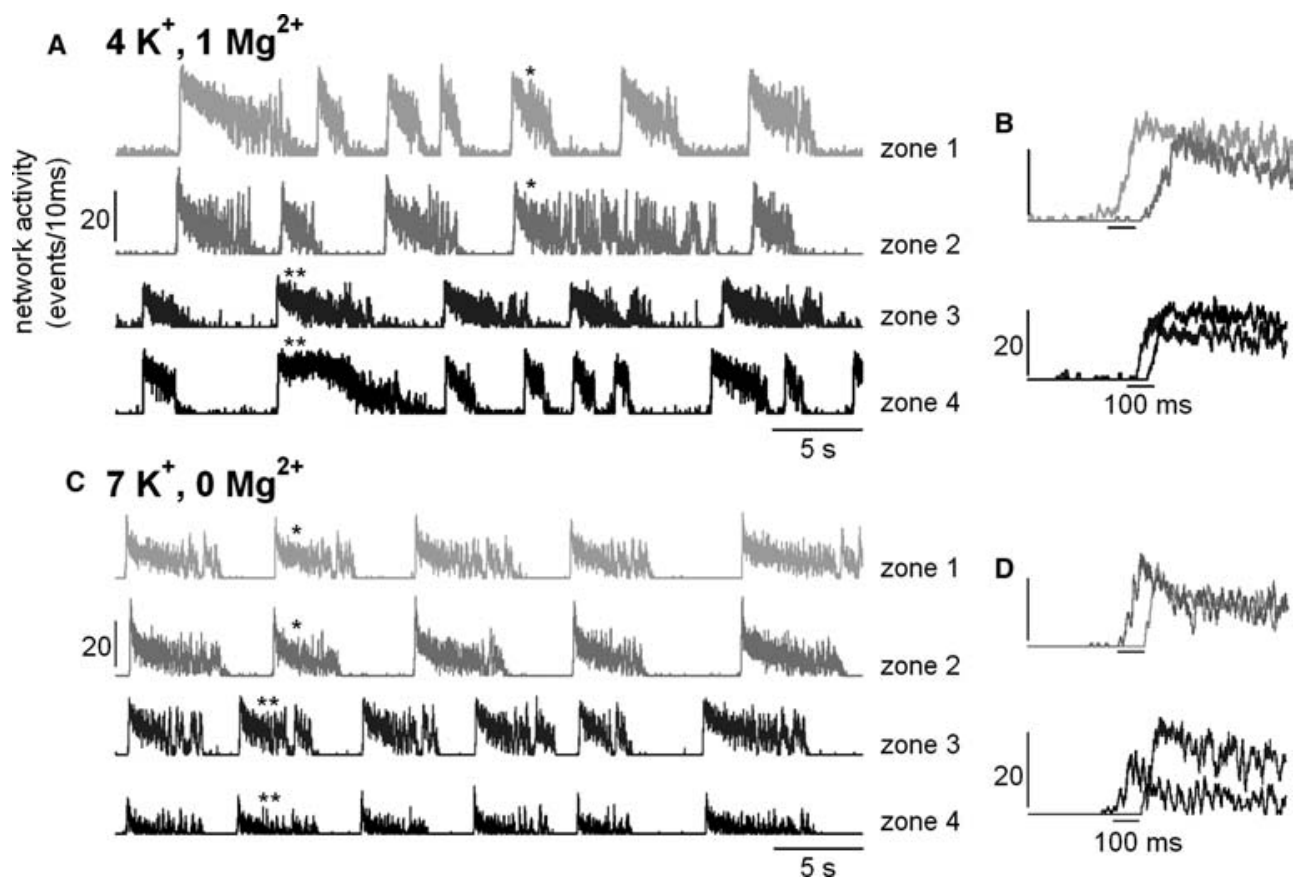

Fig. 6a-d Bursting activity in compartment cultures under control condition and conditions of increased excitability (same compartment cultures as shown in Fig. 5). a Separate network activity plots of the four zones under $4 \mathrm{mM} \mathrm{K}{ }^{+}, 1 \mathrm{mM} \mathrm{Mg}^{2+}$ b Enlarged and superimposed bursts which are correlated and marked by stars in $\mathbf{a}$. c Separate network activity plots of the four zones under low $\mathrm{Mg}^{2+}$ /high $\mathrm{K}^{+}$. d Enlarged and superimposed bursts which are correlated and marked by stars in $\mathbf{c}$. Note that despite the presence of inhibitory connections and a faster and more regular rhythm than control, low $\mathrm{Mg}^{2+} /$ high $\mathrm{K}^{+}$neither produced an alternating pattern nor increased the delays between compartments compared to disinhibition

compartments may be regulated by activity. The usual way to investigate activity-dependent modification in synaptic coupling is to compare the activity in a network (or the strength of an individual synapse) before and after application of a stimulation protocol, which is expected to induce plasticity. However, this is an almost impossible approach in spinal cultures, because the high level of spontaneous activity in such networks strongly interferes with the external stimulation. The outcome of stimulation is, therefore, variable and difficult to interpret. We have previously shown that population bursts can be triggered by external stimulation in disinhibited cultures. However, the success of stimulation in activating the network depends on many factors, such as the frequency, intensity and site of stimulation, as well as the delay from the last spontaneous burst (Darbon et al. 2002). To reliably pace the network and thus induce regular bursting, the frequency of stimulation has, therefore, to be close to the rate of spontaneous bursting. A too fast frequency leads to failures in burst stimulation, a too slow frequency leads to the occurrence of spontaneous bursts and thus to a "de-coupling" of bursting from stimulation. Failures in burst triggering are usually preceded by an increase in the delays between the stimuli and the bursts, which could last for up to
$500 \mathrm{~ms}$ and more (see Fig. 7). These delays became longer with higher frequencies of stimulation (Darbon et al. 2002). In compartment cultures, stimulation in one compartment could lead to reliable and regular bursting in the stimulated compartment, but to a de-coupling of this compartment from the neighboring one (see Fig. 7, where every third stimulus applied to the black compartment evoked a burst). Again, such de-coupling was preceded by an increase in the delays between compartments. A de-coupling of previously coupled compartments was the most frequently seen stimuli-induced modification, which persisted after stimulation. It occurred in five of six experiments in which two or more compartments were stimulated in parallel. However, with stimulation of single compartments, a coupling of previously uncoupled compartments was also seen occasionally.

As mentioned before, these inconsistent results are difficult to interpret due to the interference of external stimuli with spontaneous activity. To search for activitydependent modifications in coupling, we therefore investigated the effect of spontaneous activity on the percentage of coupled bursts and on mean delay between compartments. To do this, we either blocked spontaneous activity with TTX $(1.5 \mu \mathrm{M})$ in one group of cultures or increased it with bicuculline and strychnine $(\mathrm{B} / \mathrm{S})$ in another. Both drugs were chronically applied with the culture medium for 1-2 weeks. They were removed $24 \mathrm{~h}$ before the experiments. Then the patterns of activity either induced by disinhibition or by low $\mathrm{Mg}^{2+} /$ high $\mathrm{K}^{+}$ were compared between the two groups and a control group. As shown in Fig. 8, no difference was found between $\mathrm{B} / \mathrm{S}$ and control cultures, neither for the percentage of coupled bursts nor for the mean delay. Comparing the TTX-group with the control, we found a significantly higher percentage of coupled bursts for the 


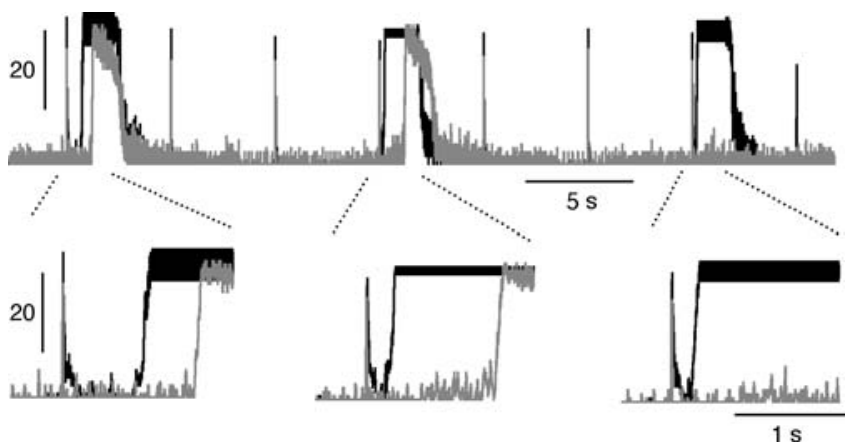

Fig. 7 Activity-dependent coupling. Network plots of two compartments in a compartment culture are superimposed (gray and black). Bursts were evoked during disinhibition by external stimulation through one MEA electrode in the black compartment. In this compartment, every third stimulus triggered a burst with variable delay. Bursts in the gray compartment were triggered by inputs from the black compartment. This coupling was unreliable with increasing delays and intermittent failures for successive bursts

low $\mathrm{Mg}^{2+} /$ high $\mathrm{K}^{+}$-evoked rhythms $(+22 \pm 7 \%, n=10$, $P=0.015)$. In contrast, we found no such difference for the disinhibition induced rhythms, suggesting that the
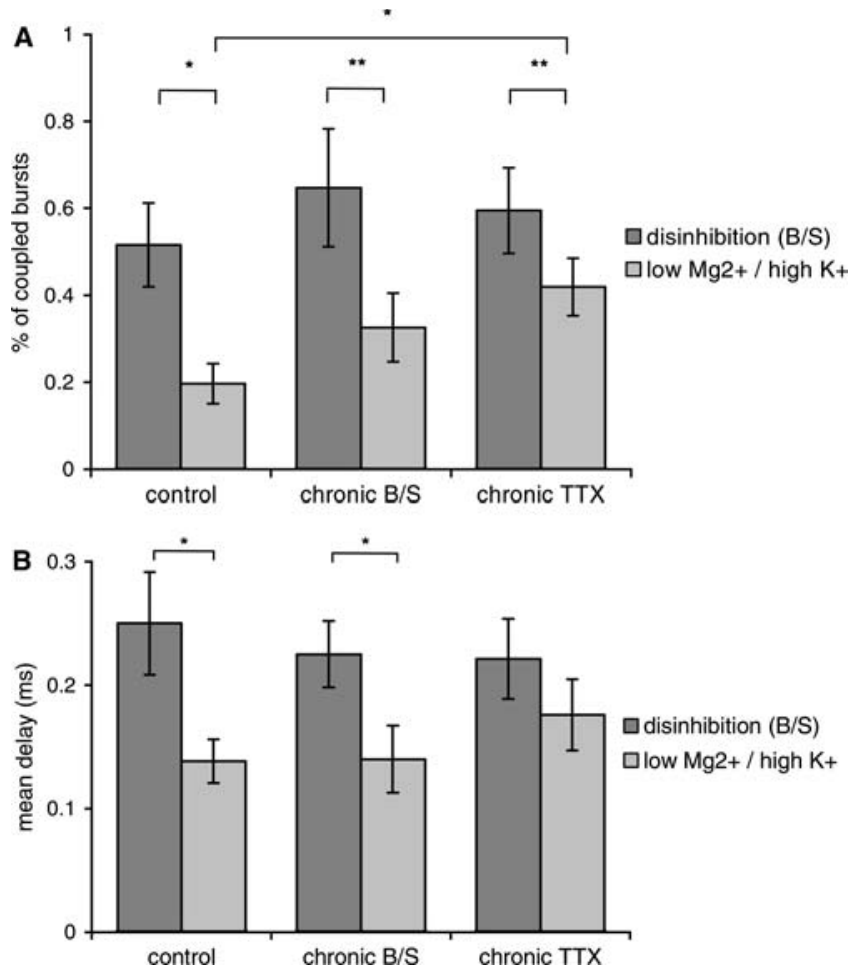

Fig. 8a, b Effects of chronic exposure to bicuculline and strychnine $(B / S)$ or TTX on the percentage of coupled bursts and mean delay in the compartment cultures. a The percentage of coupled bursts was significantly decreased under high excitability compared with disinhibition in control cultures $(n=10, P=0.012)$, by chronic exposure to $\mathrm{B} / \mathrm{S}(n=7, P=0.007)$ and by chronic exposure to TTX $(n=12, P=0.009)$. However, this decrease was partially prevented by chronic exposure to TTX compared with control $(n=10$, $P=0.015)$. b The mean delay was significantly decreased under high excitability compared to disinhibition in control cultures $(n=7$, $P=0.014)$ and $\mathrm{B} / \mathrm{S}$ treated cultures $(n=7, P=0.043)$
TTX treatment decreased the inhibitory transmission between the compartments. This is in agreement with TTX effects on other parameters of network activity such as a decrease in the burst rate under low $\mathrm{Mg}^{2+}$ / high $\mathrm{K}^{+}(-19 \pm 3 \%, n=5, P=0.042)$ resulting from prolonged bursts and intervals. This tendency could also be attributed to a lack of inhibitory connections. In summary, these results suggest that suppressing spontaneous activity reduces inhibitory synaptic transmission between compartments (or the number of inhibitory axons that cross between compartments) but leaves excitatory transmission unchanged.

\section{Discussion}

Completing our previous reports about rhythm generation in spinal cord cultures, in the present study we compare the initiation and propagation of population bursts in conventional dissociated cultures and in compartment cultures. We show that activation of the network at the onset of population bursts occurs in two steps: a first rapid activation of the trigger network initiated by a few privileged intrinsically firing neurons and a second slow activation of the rest of the network. When several such networks are loosely coupled by a few axonal connections in compartment cultures, they can recruit each other into a common bursting pattern. Such mutual recruitment often occurs with long delays, which can be more than ten-times longer than the required axonal conduction times. These delays are, however, highly variable, thus preventing the establishment of stable phase shifts in bursting between the compartments. We also found that the strength of inhibitory connections between and probably also within networks is modulated by spontaneous activity. However we found no effects of activity on excitatory connections.

\section{Trigger networks and recruitment}

The population bursting induced by disinhibition in cultures of spinal neurons is a very robust pattern of activity, which has been found in virtually all cultures investigated so far. Moreover, such rhythms are a widespread phenomenon, which is not restricted to networks of dissociated spinal neurons, but which is also found in spinal slice cultures, in the isolated spinal cord of rat, mouse, chick and other species and in many other in vitro preparations of the central nervous system, such as cortical slices or brainstem slices (Del Negro et al. 2002; Sanchez-Vives and McCormick 2000). We have, therefore, made some effort during recent years to reveal the mechanisms underlying such activity. We believe that, although the networks of dissociated spinal neurons are artificial systems, which are entirely re-grown in vitro, they offer the advantage of a simplified system, in which emergent properties of unspecific neural networks can be studied. 
Population bursting requires intrinsically spiking neurons, which are able to recruit the other cells of the network through recurrent excitation. Intrinsically spiking neurons have been detected in spinal cultures (Darbon et al. 2002; Latham et al. 2000b; Streit et al. 2001) and it has been shown in a theoretical study (Latham et al. 2000a), that the number of such cells determines the condition under which networks switch from steady firing to bursting. We found about $30 \%$ of such cells in the network (percentage of intrinsically firing cells in whole-cell recordings), which seems to be sufficient to reliably and repetitively "trigger" the network and thus induce bursting. However, only a small percentage of these cells is well enough connected to the network to initiate a population burst and thus to become a burst source. Excitatory connectivity therefore seems to be the major factor, which limits the number of burst sources. Furthermore, intrinsically spiking cells are highly excitable (see Fig. 2d) and are therefore rapidly recruited into bursting and respond with high spike rates during the bursts (see Fig. 2c, d). Thus they form a "trigger network", which more likely responds with an early activation and thus mediates the recruitment of the other, less excitable, cells. The shortest delays of the early-responding cells roughly correspond to the delays expected from the axonal conduction velocity in the cultures. The axonal conduction velocity of $0.35 \mathrm{~m} / \mathrm{s}$, estimated for our cultured neurons is comparable to that reported for commissural interneurons in the isolated spinal cord of the neonatal rat (Butt et al. 2002). The late-responding cells, however, have up to ten-times longer delays. Most of this time is probably needed to integrate incoming postsynaptic potentials to reach the threshold for action potential firing. The concept of early and late responding cells explains the large differences in delays, which could occur in neighboring electrodes. When all of the highly excitable cells in a culture are strongly interconnected, they form one distributed trigger network, as was indeed found in about half of the cultures. When these cells are not entirely interconnected, it means that they are distributed in several trigger networks per culture (usually two), which are coupled only through the late-responding less excitable cells. This explains why two or even more entirely different pathways of network activation can coexist in these cultures.

In the presence of disinhibition, NMDA induces a highly regular fast rhythm (Legrand et al. 2004). We have shown in the present work that such rhythms are always controlled by a single trigger network. The NMDA leads to depolarization of the neurons and thus increases the number of intrinsically spiking cells. Therefore, the likelihood that these cells are interconnected in one single trigger network is increased. On the other hand, the mean delay in the whole network is increased with NMDA, suggesting that the transmission from the trigger network to the rest of the network is slowed.
Minimally structured networks

We have mentioned before that the time required for the recruitment of the entire network is mostly used for the integration of incoming synaptic potentials into output spike trains. Such integration time depends on the density of synaptic inputs and the excitability of neurons. The excitability probably varies among the neurons forming the network. We expect the density of synapses to be evenly distributed in unstructured networks, although with an unknown, but probably large variation. Attempts have been made before to define the density of synapses in cultured neuronal networks by patterning the growth of the network. In the most precise case, all synaptic connections in a network are predefined by forcing the network on a pre-patterned attachment grid (Branch et al. 2000; Kleinfeld et al. 1988; Vogt et al. 2003). However, since this is a demanding approach, only small networks of a few cells have so far been constructed in such detail (Wheeler et al. 1999; Wyart et al. 2002). For larger networks, our approach of forcing the network on "macroscopic structures", and thereby influencing the density of synapses, is more realistic. Such "minimally structured" networks are still random in terms of individual synaptic contacts; however, major differences in the mean number of synapses between two defined parts of the network are introduced.

When the connections between two network pools were restricted to a few axons, as in the compartment cultures, functional separation of these networks was achieved. Such a separation either completely de-coupled the two networks, leading to an independent bursting in both pools, or, more often, bursting was synchronized in the two pools with long and variable delays. However, no qualitatively novel patterns, such as stable phase lags developed.

\section{Long delays}

How can the long delays of up to half a second be explained? Axonal conduction between compartments takes about 20-30 ms. Such short delays between compartments were occasionally seen. Nevertheless most of the delays were in the range of $150-500 \mathrm{~ms}$, suggesting that axonal conduction takes only a small part of this time. Most of it thus seems to be used for integration of incoming activity and the subsequent slow recruitment of more and more cells. We, therefore, conclude that most of the coupling between compartments was done through late-responding cells. Long delays of several hundreds of milliseconds between the stimulus and the burst onset were also observed when bursts were evoked by external stimulation (see Fig. 7). This situation is similar to coupling, since in both cases a random number of neurons in a network is activated either by the electrical stimulus or by the axonal stimuli from the neighboring network. 
Obviously recruitment is usually slow, unless a trigger network is directly activated.

During repetitive bursting an additional aspect must be considered. As shown previously, bursts are followed by a network refractory period, which is caused by a transient suppression of cellular excitability (Darbon et al. 2002). This suppression and the time-course of its relief are not evenly distributed among the neurons of a network. Assuming that the early-responding cells are those which recover faster than the late-responding cells, the latter may therefore never fully recover from such suppression of excitability during repetitive bursts, since they are permanently driven by the early-responding cells. This may contribute to their late recruitment. Using external stimulation we indeed found longer delays for higher frequencies (Darbon et al. 2002). Similar things happen when a network is driven by a faster neighbor to which it is coupled. Increasing the bursting frequency in the driving network (be it by external stimulation or by intrinsic activity) leads to an increase in the delays and finally to coupling failures (as shown in Figs. 5,7 ), equivalent to the previously shown failures in burst triggering by stimulation (Darbon et al. 2002; Legrand et al. 2004).

In a previous theoretical study, Senn et al. (1998) have shown that stable phase lags, including alternation, can arise in two weakly coupled excitatory oscillator networks. Such patterns were not seen in compartment cultures in spite of the long delays. Reasons for this probably are the large variability in the delays and the long cycle periods of population bursting, even in the presence of NMDA. Oscillation periods in the study of Senn et al. (1998) were in the range of $200-300 \mathrm{~ms}$ compared with burst periods of several seconds in the cultures. Therefore, delays of $100-500 \mathrm{~ms}$, if they are stable, may account for alternation of activity between networks during fast oscillations with frequencies of $1-$ $5 \mathrm{~Hz}$.

In the isolated spinal cord of neonatal rodents, alternate activity between the left and the right sides of the spinal cord depends on the ratio of commissural excitatory and inhibitory axons (Kiehn and Butt 2003). If this ratio is disturbed either pharmacologically (Beato and Nistri 1999) or using gene technology (Kullander et al. 2003), alternation switches to synchronous activity. In the latter study, alternation could be restored when the reciprocal inhibition was strengthened with the glycine uptake blocker sarcosine.

In compartment cultures, synchronous activity was also seen when inhibitory connections between compartments were functional (not blocked), suggesting that they were not sufficiently strong to provoke alternation. The delays between compartments were on average even shorter than during disinhibition. Strengthening the inhibitory connections with either sarcosine or the GA$\mathrm{BA}_{\mathrm{A}}$ uptake blocker nipecotic acid did not induce alternation. On the other hand, decreasing the strength of the numerous commissural excitatory axons with low doses of CNQX increased the mean delay and, at higher doses, de-coupled the compartments but never provoked stable alternation. Together these results show that, based on the randomly grown connections, it is not possible to achieve stable alternation by pharmacologically adjusting the ratio of excitatory to inhibitory transmission between the compartments.

\section{Activity-dependent modulation of coupling}

In networks of cortical or spinal neurons grown in culture, activity has been shown to cause a variety of effects, which are believed to be involved in the maintenance of homeostatic conditions in the network (Turrigiano and Nelson 2004). Nevertheless it is still an open question whether and how activity-dependent synaptic plasticity is involved in the structuring of developing networks in the spinal cord (Chen et al. 2003; Frank and Mendelson 1990). To address this question, we have investigated whether spontaneous activity can influence the percentage of coupled bursts and the delays in compartment cultures. Spontaneous activity was modified using B/S to chronically increase it and TTX to suppress it. Such treatments have been previously shown to modulate spontaneous activity in dissociated cortical cultures (Corner and Ramakers 1992; Kamioka et al. 1996). In our experiments, while $B / S$ had no effect on the percentage of coupled bursts, TTX significantly increased this percentage for bursting in the presence, but not in the absence of functional inhibitory connections. This finding suggests that less functional chloride-mediated inhibitory connections between compartments developed when activity was blocked by TTX. This hypothesis is in line with the effects of chronic TTX on other parameters, such as a decrease of the burst rate, which can also be interpreted as a lack of inhibitory connections. Blockade of spontaneous activity by TTX has been reported to depress GABAergic inhibitory synapses in spinal slice cultures (Rosato-Siri et al. 2002) and in cortical cultures (Kilman et al. 2002). In the latter, the effect of activity on GABAergic transmission is mediated by BDNF (Rutherford et al. 1997).

In summary, our results show that a loose coupling of two random networks is not sufficient to induce patterns like alternating rhythms, although large but unstable phase shifts between the rhythms in the two networks may appear. To get stable phase shifts like alternation, an appropriate ratio of crossing inhibitory and excitatory axons is probably required. Whether such more precisely structured networks can be grown in vitro using pre-patterned substrates remains to be shown.

Acknowledgements We would like to thank Denis de Limoges, Hans Ruchti, Hans-Ulrich Schweizer and Jürg Burkhalter for their technical support for the recording setup and Joseph Brader and Hans-Peter Clamann for the critical comments on the manuscript. This work was supported by the Swiss National Science Foundation with grants no. 31-59080.99 and 3100-067048.0. 


\section{References}

Ballerini L, Galante M, Grandolfo M, Nistri A (1999) Generation of rhythmic patterns of activity by ventral interneurones in rat organotypic spinal slice culture. J Physiol Lond 517:459-475

Beato M, Nistri A (1999) Interaction between disinhibited bursting and fictive locomotor patterns in the rat isolated spinal cord. J Neurophysiol 82:2029-2038

Branch DW, Wheeler BC, Brewer GJ, Leckband DE (2000) Longterm maintenance of patterns of hippocampal pyramidal cells on substrates of polyethylene glycol and microstamped polylysine. IEEE Trans Biomed Eng 47:290-300

Butt SJ, Kiehn O (2003) Functional identification of interneurons responsible for left-right coordination of hindlimbs in mammals. Neuron 38:953-963

Butt SJ, Harris-Warrick RM, Kiehn O (2002) Firing properties of identified interneuron populations in the mammalian hindlimb central pattern generator. J Neurosci 22:9961-9971

Chen HH, Hippenmeyer S, Arber S, Frank E (2003) Development of the monosynaptic stretch reflex circuit. Curr Opin Neurobiol 13:96-102

Corner MA, Ramakers GJ (1992) Spontaneous firing as an epigenetic factor in brain development - physiological consequences of chronic tetrodotoxin and picrotoxin exposure on cultured rat neocortex neurons. Brain Res Dev 65:57-64

Darbon P, Scicluna L, Tscherter A, Streit J (2002) Mechanisms controlling bursting activity induced by disinhibition in spinal cord networks. Eur J Neurosci 15:671-683

Darbon P, Tscherter A, Yvon C, Streit J (2003) Role of the electrogenic $\mathrm{Na} / \mathrm{K}$ pump in disinhibition-induced bursting in cultured spinal networks. J Neurophysiol 90:3119-3129

Del Negro CA, Morgado-Valle C, Feldman JL (2002) Respiratory rhythm: an emergent network property?. Neuron 34:821-830

Droge MH, Gross GW, Hightower MH, Czisny LE (1986) Multielectrode analysis of coordinated, multisite, rhythmic bursting in cultured CNS monolayer networks. J Neurosci 6:1583-1592

Fields RD, Yu C, Nelson PG (1991) Calcium, network activity, and the role of NMDA channels in synaptic plasticity in vitro. J Neurosci 11:134-146

Frank E, Mendelson B (1990) Specification of synaptic connections between sensory and motor neurons in the developing spinal cord. J Neurobiol 21:33-50

Grillner S (2003) The motor infrastructure: from ion channels to neuronal networks. Nat Rev Neurosci 4:573-586

Hamill OP, Marty A, Neher E, Sakmann B, Sigworth FJ (1981) Improved patch-clamp techniques for high-resolution current recording from cells and cell-free membrane patches. Pflügers Arch 391:85-100

Kamioka H, Maeda E, Jimbo Y, Robinson HP, Kawana A (1996) Spontaneous periodic synchronized bursting during formation of mature patterns of connections in cortical cultures. Neurosci Lett 206:109-112

Kiehn O, Butt SJ (2003) Physiological, anatomical and genetic identification of CPG neurons in the developing mammalian spinal cord. Prog Neurobiol 70:347-361

Kilman V, van Rossum MC, Turrigiano GG (2002) Activity deprivation reduces miniature IPSC amplitude by decreasing the number of postsynaptic $\operatorname{GABA}(\mathrm{A})$ receptors clustered at neocortical synapses. J Neurosci 22:1328-1337

Kleinfeld D, Kahler KH, Hockberger PE (1998) Controlled outgrowth of dissociated neurons on patterned substrates. J Neurosci 8:4098-4120

Kullander K, Butt SJ, Lebret JM, Lundfald L, Restrepo CE, Rydstrom A, Klein R, Kiehn O (2003) Role of EphA4 and EphrinB3 in local neuronal circuits that control walking. Science 299:1889-1892
Latham PE, Richmond BJ, Nelson PG, Nirenberg S (2000a) Intrinsic dynamics in neuronal networks I Theory. J Neurophysiol 83:808-827

Latham PE, Richmond BJ, Nirenberg S, Nelson PG (2000b) Intrinsic dynamics in neuronal networks II experiment. J Neurophysiol 83:828-835

Legrand JC, Darbon P, Streit J (2004) Contributions of NMDA receptors to network recruitment and rhythm generation in spinal cord cultures. Eur J Neurosci 19:521-532

Muller W, Swandulla D (1995) Synaptic feedback excitation has hypothalamic neural networks generate quasirhythmic burst activity. J Neurophysiol 73:855-861

Rekling JC, Feldman JL (1998) PreBotzinger complex and pacemaker neurons: hypothesized site and kernel for respiratory rhythm generation. Annu Rev Physiol 60:385-405

Robinson HP, Torimitsu K, Jimbo Y, Kuroda Y, Kawana A (1993) Periodic bursting of cultured cortical neurons in low magnesium: cellular and network mechanisms. Jpn J Physiol 43:S125-S130

Rohr S, Scholly DM, Kleber AG (1991) Patterned growth of neonatal rat heart cells in culture. Morphological and electrophysiological characterization. Circ Res 68:114-130

Rohr S, Kleber AG, Kucera JP (1999) Optical recording of impulse propagation in designer cultures. Cardiac tissue architectures inducing ultra-slow conduction. Trends Cardiovasc Med 9:173179

Rosato-Siri M, Grandolfo M, Ballerini L (2002) Activity-dependent modulation of GABAergic synapses in developing rat spinal networks in vitro. Eur J Neurosci 16:2123-2135

Rutherford LC, DeWan A, Lauer HM, Turrigiano GG (1997) Brain-derived neurotrophic factor mediates the activity-dependent regulation of inhibition in neocortical cultures. J Neurosci 17:4527-4535

Sanchez-Vives MV, McCormick DA (2000) Cellular and network mechanisms of rhythmic recurrent activity in neocortex. Nat Neurosci 3:1027-1034

Senn W, Wannier T, Kleinle J, Lüscher H-R, Müller L, Streit J, Wyler K (1998) Pattern generation by two coupled time-discrete neural networks with synaptic depression. Neural Comput 10:1251-1275

Staley KJ, Longacher M, Bains JS, Yee A (1998) Presynaptic modulation of CA3 network activity. Nat Neurosci 1:201-209

Steriade M (2001) Impact of network activities on neuronal properties in corticothalamic systems. J Neurophysiol 86:1-39

Streit J, Tscherter A, Heuschkel MO, Renaud P (2001) The generation of rhythmic activity in dissociated cultures of rat spinal cord. Eur J Neurosci 14:191-202

Tscherter A, Heuschkel MO, Renaud P, Streit J (2001) Spatiotemporal characterization of rhythmic activity in rat spinal cord slice cultures. Eur J Neurosci 14:179-190

Turrigiano GG, Nelson SB (2004) Homeostatic plasticity in the developing nervous system. Nat Rev Neurosci 5:97-107

Vogt AK, Lauer L, Knoll W, Offenhausser A (2003) Micropatterned substrates for the growth of functional neuronal networks of defined geometry. Biotechnol Prog 19:1562-1568

Wheeler BC, Corey JM, Brewer GJ, Branch DW (1999) Microcontact printing for precise control of nerve cell growth in culture. J Biomech Eng 121:73-78

Whittington MA, Traub RD (2003) Interneuron diversity series: inhibitory interneurons and network oscillations in vitro. Trends Neurosci 26:676-682

Wyart C, Ybert C, Bourdieu L, Herr C, Prinz C, Chatenay D (2002) Constrained synaptic connectivity in functional mammalian neuronal networks grown on patterned surfaces. J Neurosci Methods 117:123-131 